\title{
Evaluación de normalización de las revistas contenidas en el Índice del Consejo Nacional de Ciencia y Tecnología de México
}

\author{
Jaime Ríos Ortega *
}

Artículo recibido:

25 de abril de 2005.

Artículo aceptado:

11 de mayo de 2005.

\section{RESUMEN}

Se presentan los resultados de la evaluación practicada al Índice de Revistas Mexicanas de Investigación Científica y Tecnológica del Consejo Nacional de Ciencia y Tecnología (CONACyT), en relación con el porcentaje de normalización que muestran. Los criterios empleados se tomaron de las directrices elaboradas por el Grupo de Trabajo de Información y Documentación de la Comisión Nacional de España de la UNESCO. Los resultados indican que ninguna de las 76 revistas evaluadas alcanza más del 90\% del porcentaje de normalización. Es poco significativo el número de revistas calificadas como muy bien normalizadas ya que únicamente son 7 (9\%); 30 revistas (39\%) son aceptables en términos

* Centro Universitario de Investigaciones Bibliotecológicas de la UNAM, México. jrios@servidor.unam.mx

INVESTIGACIÓN BIBLIOTECOLÓGICA, Vol. 20, Núm. 40, enero/junio, 2006, México, ISSN: 0187-358X. pp. 121-148 
de normalización; 25 de ellas (32\%), presentan deficiencias de normalización considerables, y 14 (20\%) no son aceptables. Con base en las dos últimas cifras se aprecia que prácticamente la mitad de revistas del Índice, tienen fallas importantes de normalización. De acuerdo con los promedios obtenido por cada sección evaluada de las revistas, la sección con menor promedio fue la relacionada con "Sumario o Contenido". Importa destacar que en todos los casos existe algún tipo de problema de normalización que es posible superar.

Palabras clave: Normalización de revistas académicas; Evaluación de revistas científicas.

\section{ABSTRACT}

Assessment of journal standardization in the Index of the Mexican National Council of Science and Technology Jaime Rios-Ortega

Results of assessment on the Index of Mexican Journals of Scientific and Technological Research of the National Council on Science and Technology (CONACyT) in relation to the percentage of standardization are shown. Evaluation criteria were elaborated by the Information and Documentation Work Group of the National Spanish Commission of UNESCO. Results indicate that none of the 76 journals evaluated, reached more than $90 \%$ of normalization percentage. The number of journals qualified as very well standardized was only 7 (9\%); while $30(39 \%)$ were acceptable in terms of standardization, and $25(32 \%)$ were thought of as having considerable deficiencies, and 14 (20\%) were found not acceptable in the same terms. Based on the last two ciphers it can be appreciated that practically half of the journals in the Index include important flaws of normalization. According to the media obtained for each section assessed, the one with the least average was the section called "Summary or Content". It is important to emphasize that in all cases there exists some kind of standardization problem that can be overcome.

Keywords: Academic journal standardization; Scientific journal assessment. 


\section{INTRODUCCIÓN}

Ceún explica Garvey, el principal esfuerzo de los científicos individuales Ses manufacturar nueva información con el propósito de describir nuevos datos, formular nuevos conceptos o integrar conceptualmente los datos. Asimismo, Garvey destaca que estas contribuciones deben comunicarse de tal forma que puedan ser comprendidas y verificadas por otros científicos y entonces quizá usadas para abrir nuevos campos para futuras investigaciones. Alcanzado este punto hablaríamos de contribuciones exitosas. ${ }^{1}$ De esta manera, la comunicabilidad se convierte en un rasgo sobresaliente del producto científico e incluirá el reconocimiento de los pares como una contribución única y esencial que ha logrado éxito científico en la empresa de indagación sistemática.

Este proceso ocurre gracias al establecimiento de revistas de investigación original que llevan siglos de conformar un sistema de comunicación bien estructurado. Por ello no es casual que Meadows describa a las revistas académicas como el canal formal de comunicación más significativo de la investigación científica. ${ }^{2}$ Sin embargo, a fin de garantizar que este "canal" cumpla plenamente sus objetivos de comunicación debe ser diseñado de acuerdo con normas universales.

La aplicación de normas que deben seguirse en las publicaciones científicas, así como los problemas vinculados a la transferencia de información, ha preocupado desde hace por lo menos cuatro décadas a organismos internacionales como son la Organización de las Naciones Unidas para la Educación, la Ciencia y la Cultura (UNESCO) y la International Standardization Organization (ISO). ${ }^{3}$ No obstante, se aprecia que a pesar del tiempo transcurrido y la normatividad que al respecto se ha producido, ésta se ha incorporado de distinto modo en las normas editoriales de las revistas de conocimiento original que publican las entidades académicas del país, pues mientras que las revistas publicadas en las áreas de la vida y la materia cuentan con una tradición de uso de normas editoriales más homogénea, en las revistas publicadas en las áreas de las humanidades y las ciencias sociales esta tradición es menor.

1 William D. Garvey, Communication: The essence of Science (UK: Pergamon Press, 1979), 2.

2 Jack Meadows, "Changing Patterns in Scientific Communication", en Memoria del simposio internacional, investigación sobre la comunicación cientifica: un enfoque multidisciplinario, 2-4 de octubre de 2000 en la Ciudad de México. Comp. Margarita Almada de Ascencio, Sofía Liberman y Jane M. Russel ( México: UNAM, CUIB, Facultad de Psicología, 2002), 2.

3 Véase el "Anexo 1. Guía para la redacción de artículos científicos destinados a la publicación" y el "Anexo 2, Lista de Normas ISO Relacionadas con la Transferencia de Información”, en Helmut Grünewald, Directrices para los Directores de revistas Científicas y Técnicas ( París: UNESCO, 1982), 47-50. 
Se observan dos perspectivas que hacen necesario evaluar la normalización de las revistas académicas y elevar su calidad.

En la primera perspectiva está lo señalado por Ruiz Pérez y Pinto Molina, ${ }^{4}$ quienes mencionan que la actividad normativa ha de iniciarse en el momento en que el documento es generado, tanto en su forma física como en su contenido, y porque su práctica implica la regulación del ciclo de transferencia de información, es decir, favorece cada una de las fases del ciclo:

- La producción del documento como resultado final de la investigación.

- La transformación de la información y la generación del documento referencial cuyo fin es facilitar su control.

- La recuperación y difusión de la información para el aprovechamiento por parte del usuario.

En la segunda perspectiva el proceso de transferencia de información es afectado por diferentes variables de carácter exógeno que tienen diferente origen, a saber:

1. La necesidad de crear referentes de calidad a través de núcleos seleccionados de títulos académicos, como respuesta a un número creciente y disperso de revistas académicas de baja calidad.

2. El requerimiento de contar con instrumentos que faciliten la evaluación de las comunidades científicas y su producción.

3. La necesidad de impulsar políticas científicas nacionales y la asignación de fondos públicos para consolidar, promover y difundir la ciencia nacional y elevar la calidad de sus productos e impactos.

4. La aplicación cuidadosa de recursos económicos para la selección pertinente de publicaciones académicas.

5. El uso eficiente de espacios, almacenamiento y disponibilidad en las bibliotecas académicas o unidades de información especializadas.

A lo anterior se agrega, como factor de fuerte peso, que ante los crecientes costos y el gran número de revistas se ha generado una crisis de dimensión internacional que ha obligado a pensar en respuestas estratégicas, como las coaliciones, a fin de enfrentar este grave problema. ${ }^{5}$ Aquí de nueva cuenta la

4 Rafael Ruiz Pérez y María Pinto Molina, Directrices fundamentales para la normalización de revistas cientificas: recomendaciones destinadas a autores, directores y editores (Granada: Universidad de Granada, Grupo de Trabajo de Información y Documentación de la Comisión Nacional de España de la UNESCO, 1990), 10.

5 Duane Webster, "Emerging Responses to the Science Journal Crisis", en IFLA Journal 26, no.2 (2000): 97-102. 
calidad de la revista académica está implicada como criterio imprescindible para que la coalición sea competitiva.

El concepto de calidad de una revista es multifacético ${ }^{6}$ y es un hecho que la normalización es sólo un factor dentro de un conjunto de indicadores al evaluar la calidad de las revistas científicas.

Los bibliotecólogos tradicionalmente han valorado las revistas académicas tanto debido a requerimientos profesionales de selección y adquisición, como a razones de impacto y precio. Particularmente, la normalización de las revistas ha sido un punto fundamental que evalúan los bibliotecólogos, ya que dependiendo de la aceptación se nutren los servicios de información que proveen, o bien los índices y bases de datos que producen.

Por otra parte los investigadores llevan a cabo evaluaciones objetivas acerca de las revistas académicas a las cuales piensan enviar sus artículos y desde su formación se les orienta para ello. ${ }^{7}$ Esto es así porque publicar en revistas académicas prestigiadas representa tanto una consolidación como un avance en la carrera académica del investigador.

Los actores sociales involucrados en los procesos de transferencia de información, son varios: los editores, los proveedores de servicios de información y resúmenes y, por supuesto, los usuarios y demandantes de información científica. $^{8}$

En México, se publica un listado de títulos de revistas académicas que selecciona el Consejo Nacional de Ciencia y Tecnología desde 1994, previa evaluación de pares. A dicho listado se le ha denominado "Índice" y representa el núcleo de revistas más importantes que publican las diferentes comunidades científicas y entidades académicas del país. De acuerdo con Bazdresch, la primera convocatoria, de 1993, recibió 127 revistas para evaluación, y la correspondiente a 1997 incluyó 133 solicitudes, de las cuales sólo se aceptaron 56, es decir, el $42 \%$. ${ }^{9}$

Esta práctica institucional de evaluación de revistas académicas ya cumplió una década de trabajo y cabe señalar que los resultados de los títulos

6 Ronald Rousseau, "Journal Evaluation: Technical and Practical Issues", en Library Trends 50, no. 3 (2002): 418.

7 Fred Pyrczak, Evaluating Research in Academic Journals: A practical Guide to Realistic Evaluation, EU: Pyrczac, 1999.

8 Emilio Delgado López-Cortázar, "Incidencia de la normalización de las revistas científicas en la transferencia y evaluación de información científica”, en Revista de Neurología 25, no. 148 (1997): 1942-3.

9 Carlos Bazdresch, "El Índice de Revistas Mexicanas de Investigación Científica y Tecnológica del CONACYT", en Revistas Científicas en América Latina, Comp. Ana María Cetto y Octavio Alonso (México: ICSU, UNAM, CNACYT, FCE, 1999), 337. Lamentablemente no se ha publicado información adicional sobre el número de revistas que han presentado solicitud de evaluación para ingresar al Índice. 
aceptados en el índice son públicos y pueden consultarse en la hoja electrónica del CONACYT. ${ }^{10}$

El Comité de Evaluación de Revistas Mexicanas de Investigación Científica y Tecnológica actualizó en el año de 2000 nueve criterios a partir de los cuales realizaba la evaluación de las revistas y, en consecuencia, integraba el Índice. Los criterios, de los cuales sólo señaló el rubro, son los siguientes: ${ }^{11}$

1. Contenido

2. Calidad del contenido (composición del comité editorial, selección del material que se publica, integración de la cartera de árbitros, contribuciones y composición de la revista)

3. Antigüedad

4. Periodicidad

5. Regularidad

6. Estructura de los artículos (resumen, palabras clave, fechas de recepción, de aceptación, dirección institucional de los autores, resumen en otro idioma y colocación al principio del artículo)

7. Registro de índices internacionales

8. Registro en abstract

9. Distribución

De un modo u otro, todos estos criterios están vinculados con la normalización de la revista académica. Pero es evidente que no se establece un conjunto de estándares de normalización que integre un modelo sistematizado y exhaustivo, o por lo menos más completo en función de la normatividad que existe actualmente.

Por su parte, y refiriéndonos específicamente al contexto nacional, las revistas académicas o de investigación original en las áreas de las ciencias de la vida y la materia están regularmente vinculadas a tradiciones editoriales bien normadas y establecidas, a diferencia de las revistas académicas de ciencias

10 El primer "Índice” que publicó el CONACYT en 1994 se denominó "Índice de Revistas Científicas Mexicanas de Excelencia". El título ha tenido variaciones y actualmente se denomina como "Índice de Revistas Mexicanas de Investigación Científica y Tecnológica". A unos cuantos meses de que se publicara el Índice por primera vez, se inició una amplia discusión sobre la calidad y situación de las publicaciones científicas mexicanas y de otros países latinoamericanos. No obstante que diversos temas tuvieron de fondo problemas centrados en la normalización, esta última no fue abordada explícitamente. Los temas tratados y las recomendaciones de los foros académicos pueden verse en: Publicaciones científicas en América Latina, Comp. Ana María Cetto y Kai-Inge Hillerud (México: ICSU, UNESCO, UNAM, CIC, FCE, 1995).

11 Criterios de evaluación para el Índice de Revistas Mexicanas de Investigación Científica y Tecnológica del CONACYT, 2000. Actualización 14 de julio 2000. Consultado 11 de septiembre de 2000: 
sociales y humanidades. Por ello, no es extraño que en la literatura especializada se encuentren estudios comparativos en las áreas médicas o biológicas, ${ }^{12}$ por citar algunas, pero son casi nulos estos estudios en las revistas de humanidades y ciencias sociales, a las que sólo en años recientes les ha dado en el país la importancia que requieren. ${ }^{13}$

Comparativamente, en España la preocupación por la normalización de las revistas científicas ha dado frutos interesantes en cuanto a directrices. En primer lugar es importante mencionar al Grupo de Trabajo de Información y Documentación de la Comisión Nacional de España de la UNESCO, que en 1990 publicó las Directrices fundamentales para la normalización de revistas cientificas: recomendaciones destinadas a autores, directores y editores. ${ }^{14}$ Posteriormente, en 2001, se publicó La edición de revistas cientificas: Guía de buenos usos. ${ }^{15}$ Cabe señalar que ambos textos tienen como objetivo incidir en las revistas españolas de investigación original, pero su importancia también es considerable si se toma en cuenta el conjunto de países de habla hispana que también publican revistas académicas. Asimismo conviene decir que estos trabajos pueden entenderse como una actualización de las Directrices publicadas por la UNESCO en 1982, y una continuación del esfuerzo por divulgar y utilizar el conjunto de normas que abarcan. ${ }^{16}$

Las revistas académicas más prestigiadas sirven de modelo de trabajo a otras que hayan iniciado recientemente sus actividades de publicación. Por ello, es importante que si ya se cuenta con un núcleo que ha sido considerado como representativo y de alto valor académico de la ciencia que se produce en el país, se valore en su conjunto el cumplimiento de los estándares internacionales de normalización y que se incluyan éstos de modo sistemático en los requisitos de evaluación de las revistas de investigación original.

12 Por ejemplo, véase: Delgado López-Cozar, Emilio "ISO Standards for The Presentation of Scientific periodicals: Litle Known and Litle Used by Spanish Biomedical Journals”, Journal of Documentation 55, no.3 (1999): 288-309 y Sosa-de- Martínez, María Cristina, Alexandra Carnevale y Jorge Avendaño-Inestrillas, "Comparación de las normas para autores de revistas médicas mexicanas con las normas de Vancouver. La revista de investigación clínica 47, no. 3 (1995): 203-10.

13 Véase: Jaime Ríos Ortega, "Normalización de revistas científicas mexicanas: Campo de investigación y aportación”, Biblioteca Universitaria 3, no. 2 (2000): 85-91.

14 Rafael Ruiz Pérez y María Pinto Molina, Directrices fundamentales para la normalización de revistas cientificas: recomendaciones destinadas a autores, directores y editores (Granada: Universidad de Granada, Grupo de Trabajo de Información y Documentación de la Comisión Nacional de España de la UNESCO, 1990).

15 La edición de revistas cientificas: Guía de buenos usos, Coord. Adelaida Román Román (España: Centro de Información y Documentación Científica, CSIC, 2001). En el capítulo 3 que se refiere a Normalización, básicamente se ilustran las normas tratadas en las Directrices de Rafael Ruíz Pérez y María Pinto Molina. Asimismo ha sido escasamente abordada la normalización de revistas electrónicas.

16 Helmulth Grünewald, Directrices para los directores de revistas cientificas y técnicas (París: UNESCO, 1982). 
El estudio que aquí se presenta evalúa el porcentaje de cumplimiento de normalización que obtienen las revistas contenidas en el Índice de Revistas Mexicanas de Investigación Científica y Tecnológica del CONACYT (IRMCYT) del $2001,{ }^{17}$ e incluye un total de 76 títulos. La última actualización del índice corresponde a 2002 y respecto al índice de 2001 éste se redujo a 10 títulos. ${ }^{18}$

Cabe señalar que existe un estudio sobre algunas revistas del área de humanidades y ciencias sociales del Índice, pero de años anteriores. ${ }^{19}$

\section{MÉTOdos}

Con base en las Directrices fundamentales para la normalización de revistas científicas: Recomendaciones destinadas a autores, directores y editores, ${ }^{20} \mathrm{se}$ elaboró el instrumento de evaluación denominado "ficha de evaluación". El instrumento consta de seis secciones y cada una de ellas contiene un determinado número de unidades de normalización. Las secciones y el número de unidades de normalización de cada una de ellas son las siguientes:

Sección I: Cubierta (7 unidades)

Sección II: Información sobre la revista (10 unidades)

Sección III: Sumario o Contenido (11 unidades)

Sección IV: Identificación del artículo: sección bibliográfica (6 unidades)

Sección V: Identificación de la revista en los artículos (5 unidades)

Sección VI: Otros (4 unidades)

El total de unidades suma 43 , sin embargo únicamente se consideraron 42 para efectos de evaluación. Por lo que toca al ítem 25 "Mención de continuidad" de la ficha de evaluación, cuando aparece, sí es tomado en cuenta.

17 http://www. conacyt.mx./daic/revistas/index.html Consultado el 24 de octubre de 2001

18 http://www.conacyt.mx/dac/revistas/index.html Consultado el 5 de junio de 2004.

19 Jaime Ríos Ortega, Las revistas cientificas del subsistema de bumanidades, UNAM que aparecen en los indices del CONACYT: Estudio sobre su normalización, arbitraje y disponibilidad (Tesis de Maestría, Universidad Nacional Autónoma de México), 2000. En esta línea de trabajo, es interesante mencionar el análisis de normalización practicado a la Revista Interamericana de Bibliotecología por Chinchilla Rodríguez, Zaida. "Evaluación normativa de la Revista Interamericana de Bibliotecología”, en Revista Interamericana de Bibliotecología 23, no. 1-2 (2000): Separata.

20 Ruiz Pérez, Rafael y María Pinto Molina, Directrices fundamentales para la normalización de revistas cientificas: recomendaciones destinadas a autores, directores y editores, España: Universidad de Granada, Grupo de Trabajo de Información y Documentación de la Comisión Nacional de España de la UNEsCO, 1990. Aunque en 1995, Rafael Ruíz Pérez y Emilio Delgado López-Cózar elaboraron un modelo de evaluación de revistas científicas, los elementos esenciales de dicho modelo están contenidos en las Directrices de 1990. Véase: Rafael Ruíz Pérez y Emilio Delgado López- Cózar , "A Model for Assessing Compliance of Scientific Journals with International Standards”, en Libri 45 (1995): 145-159. 
El valor de cada unidad de normalización fue de dos puntos y cuando la información requerida por la norma se presentaba parcialmente, se asignó un punto. El puntaje máximo fue de 82 puntos que equivalió al 100\%. (Véase Anexo 2: Ficha de evaluación).

El instrumento se aplicó a los fascículos impresos de las revistas incluidas en el IRMCYT.

\section{Resultados}

Se evaluaron las 76 revistas del Índice de Revistas Mexicanas de Investigación Científica y Tecnológica del CONACYT. Ninguna de las revistas evaluadas logró un porcentaje de normalización por arriba del 90\% (Véase Anexo 3: Puntuación de las revistas por Área del IRMICYT)

Los resultados se organizaron en cinco rangos. El primer rango abarca del 80 al 89\% y en él se ubicaron siete revistas. El segundo rango va del 70 al $79 \%$ y en él se identificaron 30 revistas. El tercer rango cubre del 60 a 69\% y en él se involucraron 25 revistas. El cuarto rango abarca del 50 al 59\% e incluyó 11 revistas. El quinto rango va del 40 al 49\% y en él se incorporaron tres revistas.

La Tabla 1, "Revistas por rango de normalización”, muestra en orden decreciente los rangos, así como la categoría asociada a cada uno de ellos.

Tabla 1: Revistas por rango de normalización

\begin{tabular}{|l|c|c|}
\hline \multicolumn{1}{|c|}{ Normalización } & Rango & Revistas \\
\hline Muy bien & 80 al $89 \%$ & 7 \\
\hline Aceptable & 70 al $79 \%$ & 30 \\
\hline Deficiente & 60 a $69 \%$ & 25 \\
\hline No aceptable & 50 al $59 \%$ & 11 \\
\cline { 2 - 3 } & 40 al $49 \%$ & 3 \\
\hline
\end{tabular}

En el primer rango (80 al 89\%), la revista Computación y Sistemas obtuvo 73 puntos, que corresponden al 89\%. La revista Salud Pública en México calificó con 69 puntos, es decir, 84\%. La revista Crítica calificó con 67 puntos, que corresponden al $82 \%$, al igual que la revista Salud Mental. Por su parte la Revista Mexicana de Ciencias Geológicas y Agrociencias, así como la Revista Mexicana de Física, obtuvieron 66 puntos cada una que corresponden al 80\%.

En el segundo rango (70 a 79\%), las revistas que calificaron con el mayor puntaje fueron Gaceta Médica de México e Investigación Bibliotecológica, con 65 puntos cada una que corresponden al 79\%. Tanto la revista Chapingo 
Serie Horticultura como la revista Investigaciones Geográficas, Boletín del Instituto de Geografía, calificaron cada una con 64 puntos, esto es, el 78\%. Por su parte, las revistas Momento Económico y Estudios de Historia Novohispana sumaron 63 puntos cada una, que equivalen al 77\%. Las revista Papeles de Población, Revista de la Sociedad Química de México, Revista Mexicana de Análisis de la Conducta y Revista Internacional de Contaminación obtuvieron un puntaje de 62 puntos cada una que corresponden al 76\%. Por su parte, las revistas Archives of Medical Research, Boletín Médico del Hospital Infantil de México, Ciencias Marinas, La Revista de Investigación Clínica, Revista Fitotecnia Mexicana, Revista Mexicana de Investigación Educativa y la Revista Mexicana de Sociología, calificaron con 61 puntos cada una, lo que equivale al 74\%. Las revistas que lograron 60 puntos; es decir, el 73\%, son las cuatro siguientes: Folia Entomológica Mexicana, Gestión y Política Pública, Investigación Económica, y Política y Gobierno. Tres revistas calificaron con 59 puntos cada una, que corresponden al 72\%: Atmósfera, Geofísica Internacional y Terra. También otras tres calificaron con 58 puntos, que equivalen al 71\%, estas revistas son: Acta Zoológica Mexicana, Revista Mexicana del Caribe y "Zoología” y Anales del Instituto de Biología. Tres revistas calificaron con 57 puntos, es decir, 70\%, las revistas son: Foro Internacional, Ingeniería Hidráulica en México y Veterinaria México.

En el tercer rango del 60 a 69\%, la revista de mayor puntaje fue Escritos, Revista del Centro de Ciencias del Lenguaje que calificó con 57 puntos. Frontera Norte, Problemas del Desarrollo y Revista Internacional de Filosofía Política, obtuvieron cada una 56 puntos, que equivalen al 68\%. Únicamente el Boletín de la Sociedad Matemática Mexicana y Estudios Sociológicos de El Colegio de México calificaron con 55 puntos, que corresponden al 67\%. Las revistas: Convergencia: Revista de Ciencias Sociales, Estudios de Asia y África, Perfiles Educativos, Revista Mexicana de Ingeniería Biomédica, así como la Revista Mexicana de Fitopatología marcaron 54 puntos cada una, que equivalen al 66\%. Por su parte, la revista Historia Mexicana y la Revista Mexicana de Astronomía y Astrofísica obtuvieron 53 puntos, que corresponden al 65\%. Cuatro revistas obtuvieron 52 puntos cada una, que equivalen al 63\%; estas revistas son: "Botánica" Anales del Instituto de Biología, Economía Mexicana, Hidrobiología, así como Región y Sociedad. Revista del Colegio de Sonora. La revista Técnica Pecuaria en México calificó con 51 puntos, que equivalen al 62\%. Las revistas: Estudios Demográficos y Urbanos, Historia y Grafía, Instrumentación y Desarrollo (Instrumentation E Development), así como Sociológica, calificaron con 50 puntos cada una, que corresponden al 61\%. Tres revistas calificaron con 49 puntos cada una, que corresponden al 60\%, éstas son: Nova Tellus, Secuencia y Nueva Antropología, Revista de Ciencias Sociales. 
En el cuarto rango (50 al 59\%), las revistas que lograron mayor puntaje fueron Cuicuilco y Estudios Económicos, cada una con 48 puntos. Dos revistas alcanzaron 47 puntos, es decir, el 57\%, éstas son: Acta Botánica Mexicana y Estudios sobre las Culturas Contemporáneas. La revista El Trimestre Económico calificó con 46 puntos que equivalen al 56\%. La revista Comunicación y Sociedad obtuvo 45 puntos, que corresponden a 55\%. Cuatro revistas calificaron con 44 puntos, que equivalen al 54\%; estas revistas son: Argumentos: Estudios Críticos de la Sociedad, Boletín Mexicano de Derecho Comparado, Espiral: Estudios sobre Estado y Sociedad, así como Perfiles Latinoamericanos. La revista Anales del Instituto de Investigaciones Estéticas calificó con 43 puntos, que equivalen al $52 \%$.

En el quinto rango (40 al 49 al \%), la revista Nueva Revista de Filología Hispánica alcanzó 39 puntos, que equivalen al 47\%. La Revista Mexicana de Ciencias Políticas y Sociales calificó con 37 puntos que corresponden al 45\%. Finalmente la revista "Relaciones", Estudios de Historia y Sociedad calificó con 35 puntos que equivalen al $43 \%$.

Al promediar las calificaciones de las revistas en relación con su propia área de conocimiento de acuerdo con los puntos obtenidos, se obtienen los resultados que se muestran en la Tabla 2, "Promedio de puntuación de revistas por área”, ordenados dichos promedios en orden decreciente.

Tabla 2: Promedio de puntuación de revistas por Área

\begin{tabular}{|l|c|}
\hline \multicolumn{1}{|c|}{ Área } & Puntuación \\
\hline Área 4 Ciencias de la Tierra, del Mar y la Atmósfera & 62 \\
\hline Área 1 Ciencias Aplicadas Biológicas & 60 \\
\hline Área 2 Ciencias Aplicadas a la Ingeniería & 60 \\
\hline Área 3 Ciencias de la Salud & 60 \\
\hline Área 5 Ciencias Exactas & 59 \\
\hline Área 7 Ciencias Naturales & 54 \\
\hline Área 6 Ciencias Humanas y de la Conducta & 53 \\
\hline Área 8 Ciencias Sociales & 52 \\
\hline
\end{tabular}

Destaca con el promedio más alto el Área 4, Ciencias de la Tierra, del Mar y la Atmósfera (62 puntos) y el Área 8, Ciencias Sociales (52 puntos) con el promedio más bajo.

En la Tabla 3, "Secciones y promedios de las revistas por área de conocimiento", que se presenta a continuación, se concentran los resultados de los promedios más altos y más bajos. La coincidencia es total en la última columna en cuanto a la Sección III. 
Tabla 3: Secciones y promedios de las revistas por área de conocimiento

\begin{tabular}{|l|c|c|}
\hline \multicolumn{1}{|c|}{ Área } & $\begin{array}{c}\text { Sección / promedio } \\
\text { más alto en pts. }\end{array}$ & $\begin{array}{c}\text { Sección / promedio } \\
\text { más bajo en pts. }\end{array}$ \\
\hline Área 1 Ciencias Aplicadas Biológicas & $\mathrm{IV} / 11$ & $\mathrm{III} / 12$ \\
Área 2 Ciencias Aplicadas a la Ingeniería & $\mathrm{V} / 9$ & $\mathrm{II} / 12$ \\
Área 3 Ciencias de la Salud & $\mathrm{II} / 17$ & $\mathrm{II} / 14$ \\
Área 4 Ciencias de la Tierra, del Mar y la Atmósfera & $\mathrm{IV} / 11$ & $\mathrm{II} / 11$ \\
Área 5 Ciencias Exactas & $\mathrm{V} / 9$ & $\mathrm{II} / 10$ \\
Área 6 Ciencias Humanas y de la Conducta & $\mathrm{V} / 8$ & $\mathrm{II} / 10$ \\
Área 7 Ciencias Naturales & $\mathrm{V} / 8$ & $\mathrm{II} / 11$ \\
Área 8 Ciencias Sociales & $\mathrm{II} / 15$ & $\mathrm{II} / 10$ \\
\hline
\end{tabular}

Nota: Las Secciones en número romano son: II Información sobre la revista; III Sumario o Contenido; IV Identificación del artículo: sección bibliográfica; y v Identificación de la revista en los artículos,

Respecto al Área 1, Ciencias Aplicadas Biológicas, la sección con promedio más alto fue la sección IV (Identificación del artículo: sección bibliográfica) con 11 de 12 puntos. La más baja fue la Sección III (Sumario o Contenido) con 12 de 20 puntos.

En relación con el Área 2, Ciencias Aplicadas a la Ingeniería, la sección con promedio más alto fue la sección $\mathrm{V}$ (Identificación de la revista en los artículos) con 9 de 10 puntos. La más baja fue la Sección III (Sumario o Contenido) con 12 de 20 puntos.

En relación con el Área 3, Ciencias de la Salud, la sección con promedio más alto fue la sección II (Información sobre la revista) con 17 de 18 puntos. La más baja fue la Sección III (Sumario o Contenido) con 14 de 20 puntos.

En relación con el Área 4, Ciencias de la Tierra, del Mar y la Atmósfera la sección con promedio más alto fue la sección IV (Identificación del artículo: sección bibliográfica) con 11 de 12 puntos. La más baja fue la Sección III (Sumario o Contenido) con 11 de 20 puntos.

En el Área 5, Ciencias Exactas, la sección con promedio más alto fue la sección V (Identificación de la revista en los artículos) con 9 de 10 puntos. La más baja fue la Sección III (Sumario o Contenido) con 10 de 20 puntos.

Por lo que hace al Área 6, Ciencias Humanas y de la Conducta, la sección con promedio más alto fue la sección $\mathrm{V}$ (Identificación de la revista en los artículos) con 8 de 10 puntos. La más baja fue la Sección III (Sumario o Contenido) con 10 de 20 puntos.

Respecto al Área 7, Ciencias Naturales, la sección con promedio más alto fue la sección $\mathrm{V}$ (Identificación de la Revista en los Artículos) con 8 de 10 puntos. La más baja fue la Sección III (Sumario o Contenido) con 11 de 20 puntos.

En cuanto al Área 8, Ciencias Sociales, la sección con promedio más alto fue la sección II (Información sobre la revista) con 15 de 18 puntos. La más baja fue la Sección III (Sumario o Contenido) con 10 de 20 puntos. 


\section{DisCUSIÓN DE RESULTADOS Y CONCLUSIONES}

En primer lugar destaca que ninguna revista alcanza más allá del 90\% del porcentaje de normalización.

También llama la atención, de acuerdo con los datos obtenidos, que de las 76 revistas evaluadas únicamente siete de ellas, es decir el $9 \%$, se ubican en el primer rango del 80 a $89 \%$ de porcentaje de normalización. De igual modo, es importante mencionar que el $39 \%$ de las revistas evaluadas; esto es, 30 revistas, se encuentran en el segundo rango, que va del 70 a $79 \%$ de porcentaje de normalización. De igual forma, se hace notar que el $32 \%$ de las revistas, es decir 25 títulos, forman parte del tercer rango, que abarca del 60 a $69 \%$ de normalización. Con menos peso numérico y por ello de modo menos significativo, 11 revistas (15\%), componen el cuarto rango del 50 al 59\% de normalización.

Por último, el $4 \%$ de las revistas, es decir, tres revistas, se encuentran en el último rango, quinto, que va del 40 al $49 \%$ de normalización.

Lo anterior implica que no contamos con revistas cabalmente normalizadas en el Índice de Revistas Mexicanas de Investigación Científica y Tecnológica del CONACYT. También se constata que es poco significativo el número de revistas calificadas como muy bien normalizadas, ya que se trata únicamente de siete (11\%) del total que compone el Índice. Asimismo, se afirma que una parte considerable de ellas es aceptable en términos de normalización, esto es, 30 revistas (39\%) del Índice antes mencionado. Sin embargo, 25 revistas (el 32\%), presentan deficiencias de normalización considerables. Asimismo, las 15 revistas restantes que constituyen el 20\% del total del Índice, simplemente no son aceptables en términos de normalización. Con base en las dos últimas cifras se aprecia que el $52 \%$ de las revistas evaluadas; es decir, prácticamente la mitad de revistas del Índice, tienen fallas importantes de normalización.

Con base en los promedios obtenidos por cada sección evaluada de las revistas, se afirma que en todas las áreas, la sección con menor promedio fue la sección de "Sumario o Contenido". Esto induce a considerar que en sus propias áreas, así como las valoradas en su conjunto, las revistas del Índice presentan, sin excepción alguna, problemas en esta sección. Obviamente, las variantes de una revista a otra en esta importante sección pueden ser notorias y en algunos casos fuera de toda proporción, pero el hecho que importa destacar es que en todos los casos existe algún tipo de problema.

Por otra parte, y de acuerdo con el promedio mayor por sección evaluada, cuatro de las ocho áreas son más cuidadas sobre todo, en la Sección V: Identificación de la revista en los artículos. Estas áreas son: el Área 2, Cien- 
cias Aplicadas a la Ingeniería, el Área 5, Ciencias Exactas, el Área 6, Ciencias Humanas y de la Conducta, y el Área 7, Ciencias Naturales.

En el caso del Área 1 Ciencias Aplicadas Biológicas, así como en el del Área 4, Ciencias de la Tierra, del Mar y la Atmósfera, la sección a la que se presta mayor importancia es la Sección IV: Identificación de los artículos. Por su parte el Área 3, Ciencias de la Salud y el Área 8, Ciencias Sociales, le dan mayor atención a la Sección II: Información sobre la revista.

No obstante que estamos hablando del núcleo de revistas de mayor importancia que generan las instituciones científicas del país, se aprecia que existe un rezago notable en materia de normalización que es necesario abordar. Con base en ello, se reitera la propuesta de legislar para el país la obligación jurídica de que las entidades y los editores de las revistas académicas asuman la responsabilidad de normalizar sus publicaciones y de incorporarlas a las fuentes secundarias, como es el caso de los índices nacionales e internacionales, con el fin de garantizar los procesos de transferencia de formación científica hacia la sociedad mexicana y de vincularse aún más al entorno mundial en cuanto a transferencia de conocimientos.

Esto implica también que las entidades académicas, junto con sus editores, cobren conciencia de las implicaciones de estos esfuerzos para que se incremente la preocupación por normalizar estos importantes vehículos de comunicación de la ciencia y, en consecuencia, envíen sus revistas a los servicios de indización.

En una primera instancia la diferencia entre los puntajes promedio de las áreas científicas del Índice, respecto a su normalización, puede apreciarse como poco relevante, pero las asimetrías que se observan si se comparan las revistas una a una resultan, en los casos más extremos, totalmente drásticas ya que en la escala más baja una revista calificó en sólo 35 puntos, que equivalen al 43\% de normalización, lo que comparado al puntaje máximo de 73 puntos, es decir el 89\% de normalización, marca una distancia de 42 puntos.

Los esfuerzos de normalización deben darse en todas las áreas. Sin embargo, deberán avanzar en ello con especial énfasis el Área 8, Ciencias Sociales, seguida del Área 6, Ciencias Humanas y de la Conducta, así como el Área 7, Ciencias Naturales y el Área 5, Ciencias Exactas.

Preocupa particularmente que en todas las revistas de las áreas que forman parte del Índice, la Sección III Sumario o Contenido, presente algún problema, ya que justamente esta sección es la que proporciona información muy relevante que se incorpora a los servicios de información. Esta sección de sumario o contenido se compone de identificación del sumario, el título completo de la revista, el número de volumen y fascículos, su paginación e IS$\mathrm{SN}$, los nombres de los autores, el título completo del artículo, la mención de 
continuidad, la traducción de los títulos, la lengua original en que fue escrito el artículo, así como la paginación. A diferencia de otras secciones, todos los elementos contenidos en esta sección son fundamentales para la recuperación tanto de la revista como de los artículos. Por lo tanto, su descuido afecta considerablemente el trabajo de los autores, la entidad editora y, claramente, a la comunidad de usuarios que potencialmente se beneficia con el conocimiento nuevo que presenta la revista. Afecta también la posibilidad de incorporar todos los datos que requiere el servicio de información, que al no estar completos rompen eslabones importantes del ciclo de registro y transferencia de información.

Dado que las revistas que conforman el Índice cuentan con el reconocimiento de sus pares y de hecho cumplen con criterios de calidad esenciales para mantenerse dentro de este núcleo fundamental de revistas de investigación original, tales como el arbitraje riguroso y su visibilidad nacional e internacional, es deseable que su función como vehículos de comunicación de la ciencia no tenga obstáculos. Por ello es absolutamente indispensable garantizar que cumplan los objetivos y funciones que tienen previstos dentro de la empresa científica. Es entonces necesario no perder de vista que estos vehículos de comunicación del conocimiento original forman parte de un proceso esencial para la propia ciencia: la transferencia de información. Esto significa que debe apoyarse sin ninguna restricción, particularmente en las áreas en las que no ha existido una tradición de trabajo de normalización de revistas, todo esfuerzo orientado a que en el corto plazo se alcancen los estándares internacionales de normalización.

A la fecha, la evaluación que hace el CONACYT de las revistas a las que les interesa formar parte del Índice o mantenerse en él, incluye elementos de normalización. Sin embargo, y de acuerdo con los resultados que se presentan en este estudio, se constata que no existe una evaluación más sistemática de los elementos de normalización que se consideran esenciales, tanto en número como en forma. Por ello sería conveniente que este organismo contara con una propuesta más sistemática y exhaustiva del conjunto de elementos que son de carácter imprescindible en la normalización, con lo cual garantizaría un aspecto sustantivo de la calidad de las revistas científicas mexicanas.

En este sentido, es importante señalar que en algunas revistas el problema no es que carezcan de aquello que la norma exige; más bien, el problema es la adecuada ubicación de aquellos elementos que prescribe la norma dentro de la revista o el artículo de investigación. En otros casos es necesario incorporar el criterio editorial que exige la norma, así como su adopción y práctica. En este estudio los fascículos sobre los cuales se llevo a cabo la evaluación pueden verse en el Anexo 4: Fascículos evaluados. 
La normalización de las revistas académicas es una condición necesaria para alcanzar un adecuado proceso de transferencia de información científica en el país y el mundo, e igualmente repercute en el avance y la consolidación de las carreras de los científicos y la visibilidad de la ciencia de los países. Sin embargo la importancia que reviste este aspecto no ha logrado aún hacerse presente en la mayor parte de las revistas académicas de nuestro país.

Aunque las revistas académicas en el mundo tienden a difundirse tanto en formato impreso como electrónico, y cada vez más en éste último, las revistas mexicanas incluidas en el Índice básicamente se presentan en formato impreso, el cual digitalizan e incorporan en la WEB. Empero, el formato impreso es predominante en nuestro contexto nacional y por ello se justifica trabajar intensamente sobre los criterios de normalización que debe presentar, además de orientar y señalar los criterios que en el contexto de la publicación electrónica deben cumplirse; por supuesto, también es necesario analizar la normalización de las revistas en el formato electrónico y cómo se está desarrollando esto.

Lo anterior debe comprenderse en el contexto y las tendencias de los cambios en los modelos de comunicación científica, cuyo rasgo básico está dado por el desarrollo de la información digital y su transmisión. ${ }^{21}$ Sin embargo no puede dejar de mencionarse la reticencia que muestran algunas comunidades científicas para publicar en revistas electrónicas, a pesar de las ventajas técnicas que les son inherentes a éstas. ${ }^{22}$

Las normas que sirvieron de base para evaluar las revistas del estudio aquí presentado, son tan sólo una parte, la más sustantiva, pero sólo una porción de ellas. Como sucede con toda norma, surge por una necesidad de regular y su aplicación también obedece al uso y la ventaja que reporta dicho uso, o bien, como sucede en muchos casos, es una reacción a la incorporación de las tecnologías de información y comunicación.

Sin embargo, los problemas de normalización que se vislumbran no son derivados de la eficacia de las normas o su obsolescencia, sino de su desconocimiento. Resulta así que de no corregirse los problemas de normalización en las revistas académicas impresas, es fácilmente predecible que los problemas detectados se reiteren en el formato electrónico, en tanto no se tenga claridad sobre la importancia que detenta la existencia de la normalización y las funciones específicas que cumple el corpus que la constituye y, por tanto, nada de esto se aplique.

El CONACYT ya cumplió una década evaluando las revistas académicas del país. Esto significa que la práctica de la valoración de las revistas de

21 Meadows, Ibid.

22 Comunicacao científica, Com. Suzana Pinheiro Machado Mueller y Edilenice Jovelina Lima 
investigación se ha convertido en un trabajo instituido que ha tenido repercusiones importantes en el prestigio de las entidades de investigación que las producen, una de cuyas consecuencias ha sido contribuir a elevar la calidad de las revistas académicas.

La normalización es un elemento sustantivo de la calidad de las revistas de indagación sistemática, y aunque en otros países se considera que no debiera formar parte de la evaluación formal porque está fuera de duda su contribución a la comunicación científica, hay que impulsarla hasta alcanzar los mejores y más prestigiados estándares internacionales. También debe darse mayor impulso a la práctica de normalización, porque no debe olvidarse que las revistas académicas de mayor calidad sirven de modelo para otras revistas que apenas inician su publicación, así como a las nuevas comunidades epistémicas que buscan consolidar su institucionalización y establecer, como una condición necesaria, el canal formal de comunicación científica más importante: la revista de investigación original.

\section{OBRAS CONSULTADAS}

Bazdresch, Carlos, "El Índice de Revistas Mexicanas de Investigación Científica y Tecnológica del CONACYT", en Revistas Cientificas en América Latina, Comp. Ana María Cetto y Octavio Alonso. México: ICSU, UNAM, CNACYT, FCE, 1999. p. 337-353.

Chinchilla Rodríguez, Zaida, "Evaluación normativa de la Revista Interamericana de Bibliotecología”, en Revista Interamericana de Bibliotecología 23, no. 1-2 (2000): Separata.

Comunicacao científica, Comp. Suzana Pinheiro Machado Mueller y Edilenice Jovelina Lima Pasos. Brasilia: Departamento de Ciencia da Informaçao, Universidade de Brasilia, 2000.

Criterios de evaluación para el Índice de Revistas Mexicanas de Investigación Cientifica y Tecnológica del CONACYT, 2000. Actualización 14 de julio 2000. Consultado 11 de septiembre de 2000. http://www.conacyt.mx./daic/revistas/criterios_eval2000.html

Delgado López-Cozar, Emilio, "ISO Standards for the presentation of scientific periodicals: little known and little used by Spanish biomedical journals", en Journal of Documentation 55, no.3 (1999): 288-309.

Delgado López-Cortázar, Emilio, "Incidencia de la normalización de las revistas científicas en la transferencia y evaluación de información científica”, en Revista de Neurología 25, no. 148 (1997): 1942-3.

Garvey, William D., Communication: The essence of Science, UK: Pergamon Press, 1979. 
Grünewald, Helmut, Directrices para los Directores de revistas Cientificas y Técnicas, París: UNESCO, 1982.

http://www.conacyt.mx/daic/revistas/index.html

[Consultado el 24 de octubre de 2001].

http://www.conacyt.mx/dac/revistas/index.html

[Consultado el 5 de junio de 2004].

Meadows, Jack, "Changing patterns in scientific communication", en Memoria del simposio internacional, investigación sobre la comunicación cientifica: un enfoque multidisciplinario, 2-4 de octubre de 2000 en la Ciudad de México. Comp. Margarita Almada de Ascencio, Sofía Liberman y Jane M. Russel. México: UNAM, CUIB, Facultad de Psicología, 2002.

Pyrczak, Fred, Evaluating Research in Academic Journals: A practical Guide to Realistic Evaluation, EUA: Pyrczac, 1999.

Ríos Ortega, Jaime, Las revistas cientificas del subsistema de bumanidades, UNAM que aparecen en los indices del CONACYT: Estudio sobre su normalización, arbitraje y disponibilidad, Tesis de Maestría, Universidad Nacional Autónoma de México, 2000.

"Normalización de revistas científicas mexicanas: Campo de investigación y aportación", en Biblioteca Universitaria 3, no. 2 (2000): 85-91.

Rousseau, Ronald, "Journal evaluation: technical and practical issues" Library Trends 50, no. 3 (2002): 418.

Ruíz Pérez, Rafael y Emilio Delgado López - Cózar, "A model for assessing compliance of scientific journals with international standards ", en Libri 45 (1995): 145-159.

Ruiz Pérez, Rafael y María Pinto Molina, Directrices fundamentales para la normalización de revistas cientificas: recomendaciones destinadas a autores, directores y editores, Granada: Universidad de Granada, Grupo de Trabajo de Información y Documentación de la Comisión Nacional de España de la UNESCO, 1990.

Sosa de Martínez, María Cristina, Alexandra Carnevale y Jorge Avendaño-Inestrillas, "Comparación de las normas para autores de revistas médicas mexicanas con las normas de Vancouver", en $\mathrm{La}$ revista de investigación clínica 47, no. 3 (1995): 203-10.

Webster, Duane, "Emerging responses to the science journal crisis", en IFLA Journal 26, no.2 (2000): 97-102. 


\section{ANEXO I:}

Índice de Revistas Mexicanas de Investigación Científica y Tecnológica, 2001

\begin{tabular}{|c|c|}
\hline Nombre de la revista & Organismo que la publica \\
\hline \multicolumn{2}{|c|}{ I. Ciencias aplicadas biológicas } \\
\hline $\begin{array}{l}\text { 1. Agrociencia } \\
\text { 2. Revista Chapingo Serie Horticultura } \\
\text { 3. Revista Fitotecnia Mexicana } \\
\text { 4. Técnica Pecuaria en México } \\
\text { 5. Veterinaria-México }\end{array}$ & $\begin{array}{l}\text { Colegio de Postgraduados } \\
\text { Universidad Autónoma Chapingo } \\
\text { Sociedad Mexicana de Fitogenetica, A.C. } \\
\text { Inst. Nal. de Inv. Forestales, Agrícolas y Pecuar } \\
\text { UNAM-Facultad de Medicina, Veterinaria y Zoo }\end{array}$ \\
\hline \multicolumn{2}{|c|}{ II. Ciencias aplicadas a la ingeniería } \\
\hline $\begin{array}{l}\text { 1. Computación y Sistemas } \\
\text { 2. Ingeniería Hidráulica en México } \\
\text { 3. Instrumentation and Development/ } \\
\text { Instrumentación y Desarrollo }\end{array}$ & $\begin{array}{l}\text { Instituto Politécnico Nacional } \\
\text { Inst. Mex. de Tecnología del Agua, Semarnap } \\
\text { Sociedad Mexicana de Instrumentacion }\end{array}$ \\
\hline \multicolumn{2}{|c|}{ III. Ciencias de la salud } \\
\hline $\begin{array}{l}\text { 1. Archives of Medical Research } \\
\text { 2. Boletín Médico del Hospital Infantil de México } \\
\text { 3. Gaceta Médica de México } \\
\text { 4. La Revista de Investigación Clínica } \\
\text { 5. Salud Mental } \\
\text { 6. Salud Pública en México }\end{array}$ & $\begin{array}{l}\text { IMSS-Elsevier } \\
\text { Hospitall Infantil de México "Federico Gomez" S.S. } \\
\text { Academia Nal. de Medicina de México, A.C. } \\
\text { Inst. Nal. de La Nutrición "Salvador Zubirán" } \\
\text { Instituto Mexicano de Psiquiatría } \\
\text { Instituto Nacional de Salud Pública }\end{array}$ \\
\hline \multicolumn{2}{|c|}{ IV. Ciencias de la tierra, del mar y la atmósfera } \\
\hline $\begin{array}{l}\text { 1. "Investigaciones Geográficas" Boletín del } \\
\text { Instituto de Geografía } \\
\text { 2. Ciencias Marinas } \\
\text { 3. Geofísica Internacional } \\
\text { 4. Revista Internacional de Contaminación } \\
\text { Ambiental } \\
\text { 5. Revista Mexicana de Ciencias Geológicas } \\
\text { 6. Terra }\end{array}$ & $\begin{array}{l}\text { UNAM-Instituto de Geografía.Atmósfera } \\
\text { UNAM-Centro de Ciencias de la Atmósfera } \\
\text { ABC-Inst. de Inv. Oceanológicas } \\
\text { UNAM-Instituto de Geofísica } \\
\text { UATLAX-Inst. Nal. de Inv. Nucleares, U. Ver. } \\
\text { UNAM-Inst. de Geología y Soc. Geológica Mex. } \\
\text { Sociedad Mexicana de las Ciencias del Suelo, A.C. }\end{array}$ \\
\hline
\end{tabular}




\section{Nombre de la revista \\ 1. Boletín de la Sociedad Matemática Mexicana \\ 2. Mexicana de Física \\ 3. Revista de la Sociedad Química de México \\ 4. Revista Mexicana de Astronomía y Astrofísica}

Organismo que la publica

\section{Ciencias exactas}

Sociedad Matemática Mexicana

Sociedad Mexicana de Física

Sociedad Química de México, A.C.

UNAM-Instituto de Astronomía

\section{Ciencias humanas y de la conducta}

1. "Critica", Revista Hispanoamericana de Filosofía

2. Anales del Instituto de Investigaciones Estéticas

3. Cuicuilco, Nueva Época

4. Escritos, Revista del Centro de Ciencias del Lenguaje

5. Investigación Bibliotecológica

6. Nova Tellus

7. Nueva Revista de Filología Hispánica

8. Revista Internacional de Filosofía Política

9. Revista Mexicana de Análisis de la Conducta

10. Revista Mexicana de Investigación Educativa

11. Secuencia

12. Historia y Grafía

13. Estudio sobre las Culturas Contemporáneas
UNAM-Instituto de Investigaciones Filosóficas

UNAM-Instituto de Investigaciones Estéticas Escuela Nacional de Atropología e Historia

BUAP, Centro de Ciencias del Leng. Vicerrectoría UNAM-Centro Univ. de Inv. Bibliotecológicas UNAM-Instituto de Investigaciones Filológicas Nueva Revista de Filología Hispánica El Colegio de México, A.C.

UAM-Univ. Nal. de Educ. a Dist. Madrid España Sociedad Mexicana de Análisis de la Conducta Consejo Mex. de Investigación Educativa, A.C. Inst. de Inv. "Dr. Jose María Luis Mora" Universidad Iberoamericana Programa Cultura/CUIS/Univ. Colima

\section{Ciencias Naturales}

1. "Botánica", Anales del Instituto de Biología

2. "Zoología", Anales del Instituto de Biología

3. Acta Botánica Mexicana

4. Acta Zoológica Mexicana (Nueva Serie)

5. Folia Entomológica Mexicana

6. Hidrobiología

7. Revista Mexicana de Fitopatología
UNAM-Instituto de Biología UNAM-Instituto de Biología Instituto de Ecología, A.C. Instituto de Ecología, A.C. Sociedad Mexicana de Entomología A.C. UAM-Iztapalapa Sociedad Mexicana de Fitopatología A.C.

\section{Ciencias Sociales}

1. "Relaciones", Estudios de Historia y Sociedad

2. Argumentos

3. Boletín Mexicano de Derecho Comparado

4. Comunicación y Sociedad
El Colegio de Michoacán, A.C. UAM-Xoch/Div. de Ciencias Sociales y Hum. UNAM-Inst. de Inv. Jurídicas Univ. de Guadalajara- Centro Univ. de C.S. y Hum. 


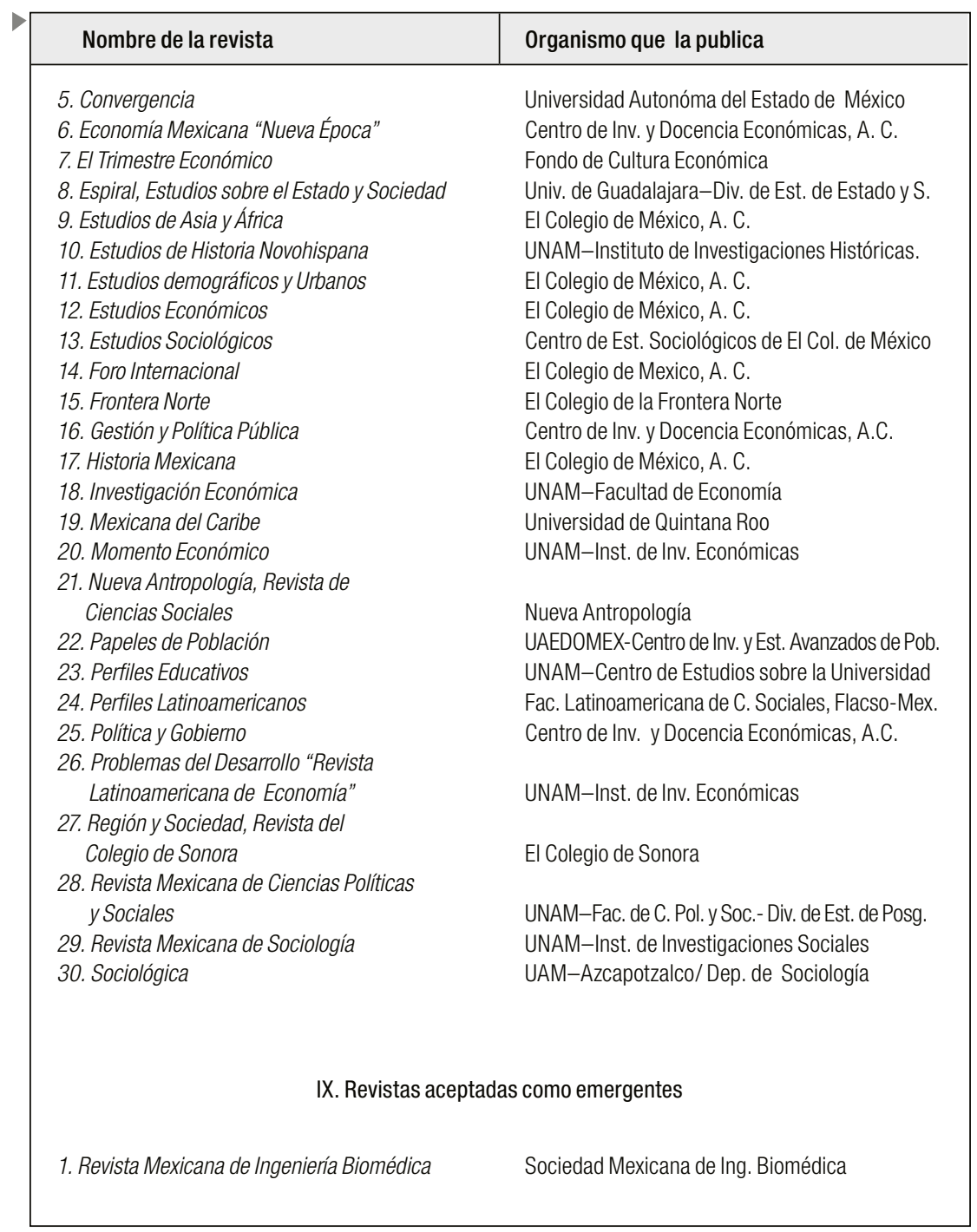


Ficha de evaluación:

Revista:

\begin{tabular}{|c|c|}
\hline Unidades de normalización & Puntuación \\
\hline \multicolumn{2}{|l|}{ I. Cubierta } \\
\hline \multicolumn{2}{|l|}{ 1. Título completo de la revista } \\
\hline \multicolumn{2}{|l|}{ 2. Numeración } \\
\hline \multicolumn{2}{|l|}{ 3. Fecha de publicación } \\
\hline \multicolumn{2}{|l|}{ 4. Lugar (de publicación) } \\
\hline \multicolumn{2}{|l|}{ 5. Nombre del editor 0 entidad responsable } \\
\hline \multicolumn{2}{|l|}{ 6. Membrete bibliográfico } \\
\hline \multicolumn{2}{|l|}{ 7. ISSN } \\
\hline \multicolumn{2}{|l|}{ II. Información sobre la revista } \\
\hline \multicolumn{2}{|l|}{ 8. Datos de la administración, redacción y director } \\
\hline \multicolumn{2}{|l|}{ 9. Integrantes del Consejo de redacción y cargos } \\
\hline \multicolumn{2}{|l|}{ 10. Integrantes del Consejo asesor } \\
\hline \multicolumn{2}{|l|}{ 11. Periodicidad y campo de cobertura } \\
\hline \multicolumn{2}{|l|}{ 12. Información para suscripción } \\
\hline \multicolumn{2}{|l|}{ 13. Depositario de derechos de autor } \\
\hline \multicolumn{2}{|l|}{ 14. No-compromiso con las opiniones de los autores } \\
\hline \multicolumn{2}{|l|}{ 15. Autorización o no para la reproducción de la revista } \\
\hline \multicolumn{2}{|l|}{$\begin{array}{l}\text { 16. Nombres de editores colaboradores y ayudas } \\
\text { para la publicación de la revista }\end{array}$} \\
\hline \multicolumn{2}{|l|}{ 17. Instrucciones para autores } \\
\hline \multicolumn{2}{|l|}{ III. Sumario o contenido } \\
\hline \multicolumn{2}{|l|}{ 18. Palabra: "sumario" o "contenido" } \\
\hline \multicolumn{2}{|l|}{ 19. Título completo de la revista } \\
\hline \multicolumn{2}{|l|}{ 20. Número del volumen y fascículo } \\
\hline \multicolumn{2}{|l|}{ 21. Paginación del fascículo } \\
\hline \multicolumn{2}{|l|}{ 22. ISSN } \\
\hline \multicolumn{2}{|l|}{ 23. Nombres de los autores } \\
\hline \multicolumn{2}{|l|}{ 24. Título completo del artículo } \\
\hline \multicolumn{2}{|l|}{ 25. Mención de continuidad } \\
\hline \multicolumn{2}{|l|}{ 26. Traducción del título entre paréntesis } \\
\hline 27. Lengua original del artículo en forma codificada & \\
\hline 28. Números de $1^{a}$ y última página separadas por un guión & \\
\hline IV. Identificación del artículo: sección bibliográfica & \\
\hline 29. Título del artículo & \\
\hline 30. Traducción del título & \\
\hline 31. Nombre(s) del autor(es) & \\
\hline 32. Fecha de recepción del original o aceptación & \\
\hline 33. Resumen en español o inglés según sea el caso & \\
\hline 34. Descriptores o palabras-clave & \\
\hline
\end{tabular}




\begin{tabular}{|c|c|c|}
\hline \multicolumn{3}{|l|}{ V. Identificación de la revista en los artículos } \\
\hline \multicolumn{3}{|l|}{ 35. Título abreviado de la revista } \\
\hline \multicolumn{3}{|l|}{ 36. Número de volumen } \\
\hline \multicolumn{3}{|l|}{ 37. Número de fascículo } \\
\hline \multicolumn{3}{|l|}{ 38. Fecha } \\
\hline \multicolumn{3}{|l|}{ 39. Páginas del artículo } \\
\hline \multicolumn{3}{|l|}{ VI. 0tros } \\
\hline \multicolumn{3}{|l|}{ 40. Mención de los servicios que indizan la revista } \\
\hline \multicolumn{3}{|l|}{ 41. Identificación institucional de los autores } \\
\hline \multicolumn{3}{|l|}{ 42. Mención de disponibilidad de la revista en formato electrónico } \\
\hline \multicolumn{3}{|l|}{ 43. Traducción de las Instrucciones para autores } \\
\hline Puntaje por sección & Obtenido & Máximo \\
\hline Subtotal sección I & & 14 \\
\hline Subtotal sección II & & 18 \\
\hline Subtotal sección III & & 20 \\
\hline Subtotal sección IV & & 12 \\
\hline Subtotal sección V & & 10 \\
\hline Subtotal sección VI & & 8 \\
\hline TOTAL & & $\begin{array}{c}82 \\
100 \%\end{array}$ \\
\hline
\end{tabular}

Fuente

Notas:

\section{ANEXO III:}

Puntuación de las revistas por Área del IRMICT*

\begin{tabular}{|c|c|c|c|c|c|c|c|c|}
\hline \multirow{2}{*}{$\begin{array}{l}\text { Área 1: Ciencias aplicadas } \\
\text { biológicas (revistas) }\end{array}$} & \multicolumn{6}{|c|}{ Secciones ${ }^{\star \star}$ y puntuación obtenida } & \multicolumn{2}{|c|}{ Total } \\
\hline & $\mathrm{I} / 14$ & II /18 & III /20 & IV/12 & $\mathrm{V} / 10$ & $\mathrm{VI} / 8$ & 82 & $100 \%$ \\
\hline Agrociencia & 12 & 14 & 13 & 12 & 7 & 8 & 66 & 80 \\
\hline Revista Chapingo Serie Horticultura & 10 & 16 & 11 & 12 & 9 & 6 & 64 & 78 \\
\hline Revista Fitotecnia Mexicana & 8 & 12 & 13 & 12 & 10 & 6 & 61 & 74 \\
\hline Veterinaria México & 10 & 14 & 11 & 10 & 8 & 4 & 57 & 70 \\
\hline Técnica Pecuaria en México & 10 & 16 & 13 & 10 & 0 & 2 & 51 & 62 \\
\hline \multirow{2}{*}{$\begin{array}{l}\text { Área 2: Ciencias aplicadas a la } \\
\text { ingeniería (revistas) }\end{array}$} & \multicolumn{6}{|c|}{ Secciones } & \multicolumn{2}{|c|}{ Total } \\
\hline & $1 / 14$ & II $/ 18$ & III /20 & IV/12 & V/ 10 & $\mathrm{VI} / 8$ & 82 & $100 \%$ \\
\hline Computación y Sistemas & 12 & 18 & 15 & 12 & 10 & 6 & 73 & 89 \\
\hline Ingeniería Hidráulica en México & 8 & 18 & 7 & 8 & 10 & 6 & 57 & 70 \\
\hline $\begin{array}{l}\text { Instrumetation \& Development I } \\
\text { Instrumentación y Desarrollo }\end{array}$ & 10 & 10 & 14 & 8 & 6 & 2 & 50 & 61 \\
\hline
\end{tabular}

* Índice de Revistas Mexicanas de Investigación Científica y Tecnológica del CONACYT (2001)

** Secciones. Sección I: Cubierta; Sección II: Información sobre la revista; Sección III: Sumario o Contenido; Sección IV Identificación del Artículo: Sección bibliográfica; Sección V: Identificación de la Revista en los Artículos; Sección VI: Otros. 


\begin{tabular}{|c|c|c|c|c|c|c|c|c|}
\hline \multirow{2}{*}{$\begin{array}{l}\text { Área 3: Ciencias de la salud } \\
\text { (revistas) }\end{array}$} & \multicolumn{6}{|c|}{ Secciones } & \multicolumn{2}{|c|}{ Total } \\
\hline & $1 / 14$ & II /18 & III /20 & $\mathrm{IV} / 12$ & $\mathrm{~V} / 10$ & $\mathrm{VI} / 8$ & 82 & $100 \%$ \\
\hline Salud Pública de México & 8 & 18 & 15 & 12 & 8 & 8 & 69 & 84 \\
\hline Salud Mental & 10 & 18 & 15 & 10 & 8 & 6 & 67 & 82 \\
\hline Gaceta Médica de México & 10 & 18 & 13 & 10 & 8 & 4 & 65 & 77 \\
\hline $\begin{array}{l}\text { Boletín Medico del Hospital } \\
\text { Infantil de México }\end{array}$ & 10 & 14 & 15 & 12 & 6 & 4 & 61 & 74 \\
\hline La Revista de Investigación Clínica & 8 & 16 & 13 & 10 & 10 & 4 & 61 & 74 \\
\hline Archives of Medical Research & 10 & 16 & 11 & 10 & 8 & 6 & 61 & 74 \\
\hline \multirow{2}{*}{$\begin{array}{l}\text { Área 4: Ciencias de la tierra, del } \\
\text { mar y la atmósfera (revistas) }\end{array}$} & \multicolumn{6}{|c|}{ Secciones } & \multicolumn{2}{|c|}{ Total } \\
\hline & $\mathrm{I} / 14$ & II /18 & III /20 & $\mathrm{IV} / 12$ & $\mathrm{~V} / 10$ & $\mathrm{VI} / 8$ & 82 & $100 \%$ \\
\hline $\begin{array}{l}\text { Revista Mexicana de Ciencias } \\
\text { Geológicas }\end{array}$ & 10 & 14 & 15 & 10 & 9 & 8 & 66 & 80 \\
\hline $\begin{array}{l}\text { Investigaciones Geográficas. Bol. } \\
\text { Inst. Geog. }\end{array}$ & 10 & 18 & 10 & 12 & 6 & 8 & 64 & 78 \\
\hline $\begin{array}{l}\text { Revista Internacional de } \\
\text { Contaminación Ambiental }\end{array}$ & 10 & 16 & 10 & 10 & 10 & 6 & 62 & 75 \\
\hline Ciencias Marinas & 8 & 14 & 12 & 12 & 10 & 6 & 62 & 74 \\
\hline Atmósfera & 12 & 14 & 13 & 8 & 6 & 6 & 59 & 72 \\
\hline Geofísica Internacional & 8 & 16 & 9 & 10 & 10 & 6 & 59 & 72 \\
\hline Terra & 10 & 14 & 11 & 12 & 8 & 4 & 59 & 72 \\
\hline \multirow{2}{*}{ Área 5: Ciencias exactas (revistas) } & \multicolumn{6}{|c|}{ Secciones } & \multicolumn{2}{|c|}{ Total } \\
\hline & $\mathrm{I} / 14$ & II/18 & $111 / 20$ & IV/12 & $\mathrm{V} / 10$ & $\mathrm{VI} / 8$ & 82 & $100 \%$ \\
\hline Revista Mexicana de Física & 10 & 18 & 12 & 10 & 10 & 10 & 6 & 80 \\
\hline $\begin{array}{l}\text { Revista de la Sociedad Química de } \\
\text { México }\end{array}$ & 10 & 16 & 10 & 10 & 10 & 6 & 62 & 76 \\
\hline $\begin{array}{l}\text { Boletín de la Sociedad Matemática } \\
\text { Mexicana }\end{array}$ & 8 & 14 & 11 & 7 & 8 & 6 & 55 & 67 \\
\hline $\begin{array}{l}\text { Revista Mexicana de Astronomía y } \\
\text { Astrofísica }\end{array}$ & 10 & 14 & 7 & 8 & 8 & 6 & 53 & 65 \\
\hline \multirow{2}{*}{$\begin{array}{l}\text { Área 6: Ciencias humanas y de la } \\
\text { conducta (revistas) }\end{array}$} & \multicolumn{6}{|c|}{ Secciones } & \multicolumn{2}{|c|}{ Total } \\
\hline & $1 / 14$ & II/18 & III /20 & $\mathrm{IV} / 12$ & $\mathrm{~V} / 10$ & $\mathrm{VI} / 8$ & 82 & $100 \%$ \\
\hline Crítica & 8 & 16 & 16 & 10 & 9 & 8 & 67 & 82 \\
\hline Investigación Bibliotecológica & 10 & 18 & 11 & 12 & 8 & 6 & 65 & 79 \\
\hline $\begin{array}{l}\text { Revista Mexicana de Análisis de la } \\
\text { Conducta }\end{array}$ & 8 & 16 & 15 & 10 & 9 & 4 & 62 & 76 \\
\hline $\begin{array}{l}\text { Revista Mexicana de Investigación } \\
\text { Educativa }\end{array}$ & 10 & 18 & 11 & 10 & 10 & 2 & 61 & 74 \\
\hline $\begin{array}{l}\text { Escritos, Revista del Centro de } \\
\text { Ciencias del Lenguaje }\end{array}$ & 10 & 18 & 9 & 6 & 10 & 4 & 57 & 69 \\
\hline $\begin{array}{l}\text { Revista Internacional de Filosofía } \\
\text { Política }\end{array}$ & 10 & 15 & 13 & 8 & 8 & 2 & 56 & 68 \\
\hline Historia y Grafía & 8 & 14 & 8 & 8 & 6 & 6 & 50 & 61 \\
\hline Nova Tellus & 10 & 9 & 11 & 10 & 7 & 2 & 49 & 60 \\
\hline Secuencia & 8 & 16 & 7 & 8 & 6 & 4 & 49 & 60 \\
\hline Cuicuilco & 10 & 14 & 8 & 6 & 8 & 2 & 48 & 59 \\
\hline
\end{tabular}




\begin{tabular}{|c|c|c|c|c|c|c|c|c|}
\hline $\begin{array}{l}\text { Estudios sobre las Culturas } \\
\text { Contemporáneas }\end{array}$ & 8 & 12 & 5 & 8 & 10 & 4 & 47 & 57 \\
\hline $\begin{array}{l}\text { Anales del Instituto de } \\
\text { Investigaciones Estéticas }\end{array}$ & 9 & 14 & 7 & 6 & 5 & 2 & 43 & 52 \\
\hline Nueva Revista de Filología Hispánica & 8 & 10 & 7 & 4 & 10 & 0 & 39 & 47 \\
\hline \multirow{2}{*}{$\begin{array}{l}\text { Área 7: Ciencias naturales } \\
\text { (revistas) }\end{array}$} & \multicolumn{6}{|c|}{ Secciones } & \multicolumn{2}{|c|}{ Total } \\
\hline & $1 / 14$ & $\| / 18$ & III /20 & IV/12 & $\mathrm{V} / 10$ & $\mathrm{VI} / 8$ & 82 & $100 \%$ \\
\hline Folia Entomológica Mexicana & 12 & 12 & 12 & 10 & 10 & 4 & 60 & 73 \\
\hline Acta Zoológica Mexicana & 12 & 12 & 10 & 10 & 8 & 6 & 58 & 71 \\
\hline $\begin{array}{l}\text { "Zoología" Anales del Instituto de } \\
\text { Biología }\end{array}$ & 10 & 14 & 13 & 8 & 9 & 4 & 58 & 71 \\
\hline Revista Mexicana de Fitopatología & 10 & 12 & 11 & 10 & 7 & 4 & 54 & 66 \\
\hline $\begin{array}{l}\text { "Botánica" Anales del Instituto de } \\
\text { Biología }\end{array}$ & 10 & 8 & 13 & 8 & 9 & 4 & 52 & 63 \\
\hline Hidrobiología & 10 & 8 & 12 & 8 & 10 & 4 & 52 & 63 \\
\hline Acta Botánica Mexicana & 10 & 8 & 10 & 10 & 7 & 2 & 47 & 57 \\
\hline \multirow[t]{2}{*}{ Área 8: Ciencias sociales (revistas) } & \multicolumn{6}{|c|}{ Secciones } & \multicolumn{2}{|c|}{ Total } \\
\hline & $1 / 14$ & II /18 & III /20 & IV/12 & $\mathrm{V} / 10$ & $\mathrm{VI} / 8$ & 82 & $100 \%$ \\
\hline Momento Económico & 10 & 16 & 10 & 12 & 7 & 8 & 63 & 77 \\
\hline Estudios de Historia Novohispana & 9 & 18 & 12 & 10 & 8 & 6 & 63 & 77 \\
\hline Papeles de Población & 8 & 16 & 11 & 6 & 5 & 6 & 62 & 76 \\
\hline Revista Mexicana de Sociología & 10 & 16 & 12 & 8 & 9 & 6 & 61 & 74 \\
\hline Gestión y Política Pública & 10 & 14 & 13 & 10 & 7 & 6 & 60 & 73 \\
\hline Investigación Económica & 10 & 14 & 11 & 10 & 9 & 6 & 60 & 73 \\
\hline Política y Gobierno & 10 & 14 & 11 & 12 & 7 & 6 & 60 & 73 \\
\hline Foro Internacional & 8 & 16 & 15 & 8 & 8 & 2 & 57 & 70 \\
\hline Revista Mexicana del Caribe & 7 & 18 & 9 & 8 & 8 & 8 & 58 & 71 \\
\hline Frontera Norte & 10 & 18 & 9 & 8 & 7 & 4 & 56 & 68 \\
\hline Problemas de Desarrollo & 10 & 18 & 11 & 6 & 7 & 4 & 56 & 68 \\
\hline $\begin{array}{l}\text { Estudios Sociológicos de El Colegio } \\
\text { de México }\end{array}$ & 8 & 14 & 13 & 12 & 6 & 2 & 55 & 67 \\
\hline $\begin{array}{l}\text { Convergencia: Revista de Ciencias } \\
\text { Sociales }\end{array}$ & 8 & 14 & 11 & 8 & 7 & 6 & 54 & 66 \\
\hline Estudios de Asia y África & 8 & 18 & 11 & 8 & 7 & 2 & 54 & 66 \\
\hline Perfiles Educativos & 10 & 16 & 7 & 8 & 9 & 4 & 54 & 66 \\
\hline Historia Mexicana & 8 & 16 & 11 & 6 & 10 & 2 & 53 & 65 \\
\hline Economía Mexicana "Nueva Época" & 10 & 12 & 11 & 6 & 7 & 6 & 52 & 63 \\
\hline $\begin{array}{l}\text { Región y Sociedad. Revista del } \\
\text { Colegio de Sonora }\end{array}$ & 10 & 10 & 11 & 8 & 7 & 6 & 52 & 63 \\
\hline Estudios Demográficos y Urbanos & 8 & 16 & 13 & 8 & 1 & 4 & 50 & 61 \\
\hline Sociológica & 10 & 12 & 7 & 8 & 9 & 4 & 50 & 61 \\
\hline $\begin{array}{l}\text { Nueva Antropología Revista de } \\
\text { Ciencias Sociales }\end{array}$ & 8 & 16 & 11 & 12 & 0 & 2 & 49 & 60 \\
\hline Estudios Económicos & 8 & 16 & 9 & 8 & 1 & 6 & 48 & 59 \\
\hline El Trimestre Económico & 12 & 13 & 10 & 8 & 1 & 2 & 46 & 56 \\
\hline Comunicación y Sociedad & 10 & 16 & 10 & 6 & 7 & 6 & 45 & 55 \\
\hline
\end{tabular}




\begin{tabular}{|l|c|c|c|c|c|c|c|c|}
\hline $\begin{array}{l}\text { Argumentos: Estudios Críticos de la } \\
\text { Sociedad }\end{array}$ & 10 & 12 & 5 & 8 & 5 & 4 & 44 & 54 \\
\hline $\begin{array}{l}\text { Boletín Mexicano de Derecho } \\
\text { Comparado }\end{array}$ & 8 & 10 & 7 & 8 & 9 & 2 & 44 & 54 \\
\hline $\begin{array}{l}\text { Espiral: Estudios sobre Estado y } \\
\text { Sociedad }\end{array}$ & 8 & 10 & 9 & 6 & 7 & 4 & 44 & 54 \\
\hline Perfiles Latinoamericanos & 8 & 14 & 7 & 8 & 5 & 2 & 44 & 54 \\
\hline $\begin{array}{l}\text { Revista Mexicana de Ciencias } \\
\text { Políticas y Sociales }\end{array}$ & 8 & 12 & 7 & 6 & 0 & 4 & 37 & 45 \\
\hline $\begin{array}{l}\text { "Relaciones" Estudios de Historia y } \\
\text { Sociedad }\end{array}$ & 8 & 12 & 7 & 6 & 0 & 2 & 35 & 43 \\
\hline $\begin{array}{l}\text { Área 9: Revistas aceptadas como } \\
\text { emergentes }\end{array}$ & $\mathrm{I} / 14$ & $\mathrm{II} / 18$ & $\mathrm{III} / 20$ & $\mathrm{IV} / 12$ & $\mathrm{~V} / 10$ & $\mathrm{VI} / 8$ & 82 & $100 \%$ \\
\hline $\begin{array}{l}\text { Revista Mexicana de Ingeniería } \\
\text { Biomédica }\end{array}$ & 10 & 10 & 13 & 10 & 9 & 2 & 54 & 66 \\
\hline
\end{tabular}

\section{ANEXO IV:}

\begin{tabular}{|c|c|}
\hline Áreas & Revista \\
\hline \multirow{5}{*}{$\begin{array}{l}\text { Área 1: Ciencias aplicadas } \\
\text { biológicas }\end{array}$} & Agrociencia 35,no.2 (2001) \\
\hline & Revista Chapingo Serie Horticultura 6, no. 2 (2000) \\
\hline & \begin{tabular}{|l} 
Revista Fitotecnia Mexicana 25, no. 1 (2002) \\
\end{tabular} \\
\hline & Técnica Pecuaria en México 38, no. 3 (2002) \\
\hline & Veterinaria México 32, no. 2 (2001) \\
\hline \multirow{3}{*}{$\begin{array}{l}\text { Área 2: Ciencias aplicadas a la } \\
\text { ingenieria }\end{array}$} & Computación y Sistemas 5, no. 1 (2001) \\
\hline & Ingeniería Hidráulica en México 15, no. 2 (2000) \\
\hline & Instrumentation \& Development 4, no. 5 (2000) \\
\hline \multirow[t]{6}{*}{ Área 3: Ciencias de la salud } & Archives of Medical Research 32, no. 4 (2001) \\
\hline & Bóletín Médico del Hospital Infantil de México 58, no. 9 (2001) \\
\hline & Gaceta Médica de México 137, no. 5 (2001) \\
\hline & La Revista de Investigación Clínica 53, no. 4 (2001) \\
\hline & Salud Mental 24, no. 4 (2001) \\
\hline & Salud Pública de México 43, no. 4 (2001) \\
\hline \multirow{7}{*}{$\begin{array}{l}\text { Área 4: Ciencias de la tierra, el mar } \\
\text { y la atmosfera }\end{array}$} & Atmósfera 14, no. 3 (2001) \\
\hline & Ciencias Marinas 24, no. 3 (2001) \\
\hline & Geofísica Internacional 34, no. 4 (1999) \\
\hline & Investigaciones Geográficas 45, (Agosto 2001) \\
\hline & Revista Internacional de Contaminación Ambiental 17, no. 1 (2001) \\
\hline & Revista Mexicana de Ciencias Geológicas 18, no. 1 (2001) \\
\hline & Terra 18, no. 4 (2000) \\
\hline \multirow[t]{4}{*}{ Área 5 ciencias exactas } & Boletín de la Sociedad Matemática Mexicana 7, no. 1 (2001) \\
\hline & Revista de la Sociedad Química de México 45, no. 3 (2001) \\
\hline & Revista Mexicana de Astronomía y Astrofísica 10, (Marzo 2001) \\
\hline & Revista Mexicana de Física 47, no. 3 (2001) \\
\hline
\end{tabular}




\begin{tabular}{|c|c|}
\hline \multirow{12}{*}{$\begin{array}{l}\text { Área 6: Ciencias humanas y de la } \\
\text { conducta }\end{array}$} & Crítica Revista Hispanoamericana de filosofía 33, no. 98 (2001) \\
\hline & $\begin{array}{l}\text { Cuicuilco Revista de la Escuela Nacional de Antropología e Historia } \\
7, \text { no. } 18(2000)\end{array}$ \\
\hline & $\begin{array}{l}\text { Escritos Revista del Centro de Ciencias del Lenguaje 22, (Julio- } \\
\text { Diciembre-2000) }\end{array}$ \\
\hline & Estudios Sobre las Culturas Contemporáneas 6, no. 12 (2000) \\
\hline & Historia y Grafía 16, (2001) \\
\hline & Investigación Bibliotecológica 16, no. 32 (2002) \\
\hline & Nova Tellus 20, no. 1 (2002) \\
\hline & Nueva Revista de Filología Hispánica 49, no. 1 (2001) \\
\hline & Revista de Filosofía Política no. 17 (Julio-2001) \\
\hline & Revista Mexicana de Análisis de la Conducta 26, no. 2 (2002) \\
\hline & Revista Mexicana de Investigación Educativa 5, no. 9 (2000) \\
\hline & Secuencia no. 48 (sep-2000) \\
\hline \multirow[t]{8}{*}{ Área 7: Ciencias naturales } & Acta Botánica Mexicana no. 55 (Agosto-2001) \\
\hline & Acta Zoológica Mexicana no. 82 (Abril-2001) \\
\hline & Anales del Instituto de Biología Serie Zoología 72, no. 1 (2001) \\
\hline & Anales del Instituto de Biología Serie Botánica 72, no. 1 (2001) \\
\hline & $\begin{array}{l}\text { Anales del Instituto de Investigaciones Estéticas 21, no. 74-75 } \\
\text { (primavera- Otoño 1999) }\end{array}$ \\
\hline & Folia Entomológica Mexicana 40, no. 2 (2001) \\
\hline & Hidrobilógica 10, no. 2 (2000) \\
\hline & Revista Mexicana de Fitopatología 18, no. 1 (2000) \\
\hline \multirow[t]{23}{*}{ Área 8: Ciencias sociales } & Argumentos Internacionales ${ }^{*}$ \\
\hline & Argumentos no. 38 (Abril-2001) \\
\hline & Boletín Mexicano no.34 (sep.-dic. 2001) \\
\hline & Comunicación y Sociedad no.35 (enero-junio 1999) \\
\hline & Convergencia no. 24 (enero-abril 2001) \\
\hline & Economía Mexicana: Nueva Época 10, no. 1 (2001) \\
\hline & El trimestre Económico 58, no.271 (2001) \\
\hline & Espiral 7, no.21 (2001) \\
\hline & Estudios de Asia y África 35, no. 3 (2000) \\
\hline & Estudios de Historia Novohispana 25, no. 25 (2001) \\
\hline & Estudios Demográficos y Urbanos 16, no. 2 (2001) \\
\hline & Estudios Económicos 16, no.1 (2001) \\
\hline & \begin{tabular}{|l} 
Estudios Sociológicos 19, no. 55 (2001) \\
\end{tabular} \\
\hline & Foro Internacional 41, no. 3 (2001) \\
\hline & Frontera Norte 13, no.24 (2000) \\
\hline & Gestión y Política Pública 10, no. 1 (2001) \\
\hline & Historia Mexicana 1, no.1 (2000) \\
\hline & Investigación Económica no. 236 (abril -junio 2001) \\
\hline & Momento Económico no.119(Enero-Febrero 2002) \\
\hline & Nueva Antropología: Revista de Ciencias Sociales 28, no.59 (2001) \\
\hline & Papeles de Población 7, no. 27 (2001) \\
\hline & Perfiles Educativos 24, no. 95(2002) \\
\hline & Perfiles Latinoamericanos 9, no, 17 (2000) \\
\hline
\end{tabular}




\begin{tabular}{|c|c|}
\hline \multirow[t]{8}{*}{ 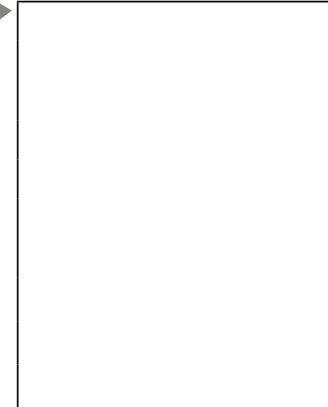 } & Política y Gobierno 8, no. 2 (2001) \\
\hline & $\begin{array}{l}\text { Problemas del Desarrollo: Revista de Latinoamerica 32, no. } 124 \\
\text { (2001) }\end{array}$ \\
\hline & Región y Sociedad: Revista del Colegio de Sonora 14, no. 23 (2002) \\
\hline & Relaciones Estudios de Historia y Sociedad 22, no.85 (2001) \\
\hline & $\begin{array}{l}\text { Revista Mexicana de Ciencias Políticas y Sociales no.179 (Mayo- } \\
\text { Agosto 2000) }\end{array}$ \\
\hline & Revista Mexicana de Sociología 64, no.2 (2002) \\
\hline & Revista mexicana del Caribe 5, no. 9 (2000) \\
\hline & Sociológica* \\
\hline Área 9: Revistas emergentes & Revista Mexicana de Ingeniería Biomédica 22, no. 1 (2001) \\
\hline \multicolumn{2}{|c|}{ Revistas fuera del índice 200, respecto al índice 2000} \\
\hline Área 2 & Instrumentation \& Development \\
\hline \multirow[t]{3}{*}{ Área 3} & Bóletín Médico del Hospital Infantil de México 58, no. 9 (2001) \\
\hline & Gaceta Médica de México 137, no. 5 (2001) \\
\hline & La Revista de Investigación Clínica 53, no. 4 (2001) \\
\hline Área 6 & $\begin{array}{l}\text { Anales del Instituto de Investigaciones Estéticas 21, no. 74-75 } \\
\text { (Primavera- otoño 1999) }\end{array}$ \\
\hline \multirow[t]{4}{*}{ Área 8} & Momento Económico no.119(Enero-febrero 2002) \\
\hline & Problemas del Desarrollo: Revista de Latinoamerica 32, no. 124 (2001) \\
\hline & Sociológica* \\
\hline & Comunicación y Sociedad no.35 (Enero-junio 1999) \\
\hline
\end{tabular}

